
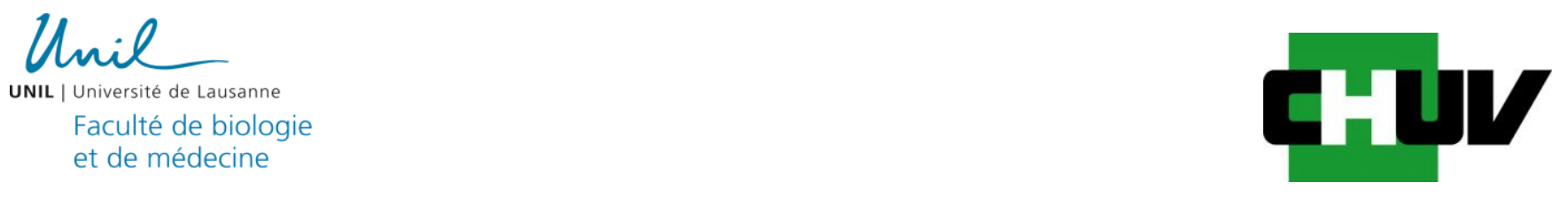

Mémoire de Maîtrise en médecine No 82

\title{
Newer antiepileptic drugs in the treatment of status epilepticus: impact on prognosis
}

Etudiante

Léonore Jaques

\author{
Tuteur \\ Dr. Andrea O. Rossetti, PD, MER \\ Dpt des neurosciences cliniques, CHUV
}

\section{Expert}

Pr Thierry Buclin

Division de pharmacologie et toxicologie clinique, CHUV

Lausanne, 05.12.2011 


\title{
Newer antiepileptic drugs in the treatment of status epilepticus: impact on prognosis
}

\author{
Léonore Jaques, BM ; Andrea O. Rossetti, MD
}

Department of Clinical Neurosciences, University Hospital and Faculty of Biology and Medicine, Lausanne, Switzerland

\author{
Contents: \\ Title: 85 characters \\ Abstract: 247 words \\ Text: 1590 words \\ 5 Tables \\ 36 References
}

Acknowledgment: The authors thank Dr Malin Maeder-Ingvar, and the EEG fellows and technologists for their help in data collection.

Address correspondence to:

Dr Andrea O. Rossetti

Service de Neurologie

CHUV-BH07

CH-1011 Lausanne, Switzerland

Phone: +4121314 1220

Fax: $\quad$ +4121314 1290

andrea.rossetti@chuv.ch 


\section{Summary}

Background: Newer antiepileptic drugs (AED) are increasingly prescribed, and seem to have a comparable efficacy as the classical AED, but are better tolerated. Very scarce data exist regarding their prognostic impact in patients with status epilepticus (SE). We therefore analyzed the evolution of prescription of newer AED between 2006-2010 in our prospective SE database, and assessed their impact on SE prognosis.

Methods: We found 327 SE episodes occurring in 271 adults. The use of older versus newer AED (levetiracetam, pregabalin, topiramate, lacosamide) and its relationship to outcome (return to clinical baseline conditions, new handicap, or death) were analyzed. Logistic regression models were applied to adjust for known SE outcome predictors.

Results: We observed an increasing prescription of newer AED over time (30\% of patients received them at the study beginning, vs. $42 \%$ towards the end). In univariate analyses, patients treated with newer AED had worse outcome than those treated with classical AED only ( $19 \%$ vs $9 \%$ for mortality; $33 \%$ vs $64 \%$ for return to baseline, p<0.001). After adjustment for etiology and SE severity, use of newer AED was independently related to a reduced likelihood of return to baseline $(\mathrm{p}<0.001)$, but not to increased mortality.

Conclusion: This retrospective study shows an increase of the use of newer AED for SE treatment, but does not suggest an improved prognosis following their prescription. Also in view of their higher price, well-designed, prospective assessments analyzing their impact on efficacy and tolerability should be conducted before a widespread use in SE.

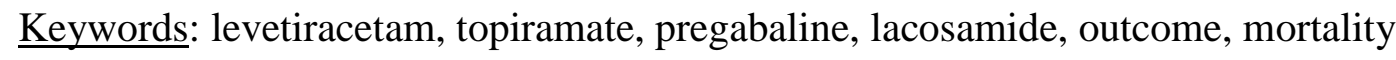


Status epilepticus (SE) represents a common neurological emergency that is associated with significant mortality and morbidity (Lowenstein, 1999), and thus requires a prompt management to avoid neurological sequelae or death (Lowenstein \& Alldredge, 1998; Shorvon, 2001).

Current SE treatment protocols advocate a three-step approach, with benzodiazepines as initial therapy followed by the use of antiepileptic drugs (AED) administrated intravenously (Meierkord et al., 2010). When SE is refractory to the two first treatment lines, a coma induction with an anaesthetic agent may be necessary (Lowenstein, 1998; Meierkord et al., 2010). Newer AED have been increasingly marketed since about two decades; globaly, efficacy seems unchanged as compared to traditional AED, but they are generally better tolerated (Kwan \& Brodie, 2003). These compounds are increasingly prescribed, although indications other than epilepsy seem to contribute to this trend (Alacqua et al., 2009). Newer AED have been used in the treatment of SE in the last years, both in oral (Towne et al., 2003; Rossetti \& Bromfield, 2006; Novy \& Rossetti, 2010; Stojanova \& Rossetti, 2011), and intravenous forms (Berning et al., 2009; Rüegg et al., 2008; Knake et al., 2008; Kellinghaus et al., 2011). It remains however unclear if the use of newer AED has lead to an improved prognosis in patients suffering from SE, and whether they are increasingly used in this setting.

The aim of this study was to describe the evolution of prescription of newer AED in SE treatment over the years 2006-2010 in our hospital, and to investigate their impact on SE prognosis.

\section{Methods}

\section{Database, patients, SE definition}

Our prospective SE database (Novy \& Rossetti, 2010) that identifies adults with SE episodes admitted to our center (a University hospital) was retrospectively analysed for this study; the period considered spans between April 1, 2006 and September 30, 2010. Patients were identified by 2 epileptologists and the neurologic consulting team; EEG were systematically performed for every patient with a suspicion of SE. Status epilepticus was considered as an epileptic seizure lasting more than 30 minutes, or several epileptic seizures without return to 
baseline between seizures. Patients under 16 years and episodes of post-anoxic SE were not included due to markedly different prognostic implications.

\section{Definition of variables}

Age, gender, history of previous seizures, seizure types (partial vs. generalized), level of consciousness, SE duration, time to treatment, treatment type and etiology of SE were prospectively identified. Older age, extent of consciousness impairment, and acute symptomatic etiology have been consistently shown to be independently related to poor outcome following SE (Logroscino et al., 1997; Towne et al., 1994; Chin et al., 2004; Claassen and al., 2002; Schneker \& Fountain, 2003; Rossetti et al., 2006); the SE severity score (STESS), a validated clinical prognostic tool considering age, seizure type, consciousness impairment, and history of previous seizures (as etiology surrogate), was prospectively calculated for each patient on admission and dichotomized in the database as $<3$ (favourable) or $\geq 3$ (unfavourable) (Rossetti et al., 2008). Etiology was considered as "potentially fatal", in analogy to previous works, if leading to death without an appropriate treatment independently of SE (Rossetti et al., 2006; Novy et al., 2010). These etiologies include for example: massive ischemic and hemorrhagic stroke, primary or secondary cerebral tumor, central nervous system (CNS) infection, severe autoimmune disease, AIDS with CNS complication, and metabolic disturbance sufficient to cause coma, eclampsia, and sepsis.

Use of AED for SE treatment was prospectively recorded in the database; for the purpose of this analysis, topiramate, levetiracetam, pregabalin and lacosamide were considered as newer AEDs ; in fact, other newer AED (such as lamotrigine, gabapentine, felbamate, vigabatrine, tiagabine, oxcarbazepine, or retigabine) were practically never used in a SE setting in our hospital. Classical AED mainly used in SE in our database are phenytoin, valproate, barbiturates, and benzodiazapines. SE episodes were dichotomized into those with at least one newer AED (irrespective of the medication) or not. Outcome at hospital discharge was prospectively categorized as return to baseline clinical conditions, disabled (i.e., presence of a new handicap), or death.

\section{Statistical analysis}

The prevalence of use of newer AED, and clinical prognosis, were first described according to two periods, defined by the availability of intravenous formulations of levetiracetam and 
lacosamide in our hospital (April 1, 2006 to September 30, 2007; October 1, 2007 to September 30, 2010). Subsequently, we analyzed outcome distribution according to the use of newer AED, and robust SE outcome predictors (etiology, STESS). Frequency tables were assessed using $\chi^{2}$ tests. Finally, stepwise multivariable logistic regressions were used to evaluate the impact of use of newer AED on prognosis, adjusting for other predictors; models were evaluated with the Hosmer-Lemeshow test. All calculations were performed with the version 9 of the Stata software (College Sattion, TX, USA); significance was considered at $\mathrm{p}<0.05$.

\section{Results}

We identified 327 SE episodes occuring in 271 patients during the 54-months study period. Mean age was 60 years $(\mathrm{SD} \pm 18.3)$. There were 176 women $(53.8 \%)$; 40 patients $(14.8 \%)$ presented more than one SE episode. Over the 5-year period, 128 SE episodes (39.2\%) were treated with at least one of the newer AED, 70 of them were women (54.7\%), and mean age in this group was 59 years ( $\mathrm{SD} \pm 16.7$; range 16-92). We observed a trend favouring the use of newer AED during the second period of time (102 episodes, 42.2\%) over the first one (26 episodes, $30.6 \%)(\mathrm{p}=0.060)($ Table 1$)$.

Prognosis according to the two chronological periods showed non-significant differences, with a slightly higher mortality in the earlier period $(\mathrm{p}=0.303)$ (Table 2). However, in univariable analyses, patients treated with newer AED had a worse outcome than patients receiving only classical AED during the entire study period (Table 3).

In the studied cohort, patients with a potentialy fatal etiology were more often treated with newer AED. There was also a trend to treat patients with a high STESS ( $\geq 3-6)$ more often with newer AED (Table 4). After adjustment for potentially fatal etiology and SE severity score (that included age, seizure semiology, extent of consciousness impairment, and history of previous seizures), use of newer AED was significantly related to a higher risk of lack of return to baseline clinical conditions at discharge, but not to inscreased mortality. Both models showed an acceptable goodness of fit, and confirmed the robustness of STESS and etiology as independent outcome predictors (Table 5). 


\section{Discussion}

This study focusing on the prescription of newer AED in patients with SE shows that their use tended to increase over the past 5 years. Moreover, treatment of SE with newer AED was associated with increased disability at hospital discharge, but not higher mortality.

A similar trend of increased prescription of newer AED has been already shown in recent studies (Alacqua et al., 2009; Savica et al., 2007); although that seemed to at least partly reflected extra-epileptic indications (e.g., neuropathic pain), other studies demonstrate that newer AED are increasingly used in patients with epilepsy (Pugh et al., 2008); however, the speed of this rise appears relatively modest (Hsia et al., 2010). To the best of our knowledge, a similar analysis has not been yet performed in patients with SE, and confirms the global trend.

In univariable analyses, patients who only received classical AED had a more favorable prognosis than those who also received newer AED $(\mathrm{p}<0.001)$. This differs from previous assessments on patients with epilepsy, which globally showed similar efficacy with fewer side effects for the newer AED (Beydoun, 1997; Kwan \& Brodie, 2003; Vasquez, 2004; Marson et al., 2007), and may reflect some confounding in our cohort. In fact, consideration of known SE outcome predictors discloses that patients with a potentialy fatal etiology (Rossetti et al., 2006; Tsai et al., 2009) had received newer AED more often for the treatment of their SE episode ( $\mathrm{p}=0.001$ ); furthermore, subjects with a high STESS $(\geq 3)$ (Rossetti et al., 2008) also tended to show a higher chance to be treated with newer AED $(\mathrm{p}=0.057)$. These two variables thus may explain at least in part the results: patients with SE forms felt to be more aggressive had a greater likelihood to receive further compounds of the therapeutic arsenal, including newer AED; this is highlighted by the multivariable analysis: after correction for etiology and SE severity, only disability, but not mortality, resulted independently related to the use of newer AED. A recent study analysing the same database showed a higher rate of SE treatment failure with levetiracetam than with valproate but no impact on the outcome at hospital discharge (Alvarez et al., 2011). A possible explanation for these results could be that dosages of levetiracetam used in our hospital, between 1000-3000 mg daily, were somewhat lower than those recently recommended in the literature (3,000-3,500 mg daily) (Berning et al., 2009; Trinka \& Dobesberger, 2009; Shorvon, 2011; Swisher et al., 2011). 
While a greater risk of disability after use of newer AED could theoretically reflect some reduced efficacy of these compounds, an information bias may also exist regarding the outcome. Indeed, the "return to baseline" clinical conditions may depend on the moment it was assessed, as disability may evolve over time, as opposed to mortality that represents a more "robust" outcome. In our database, outcome was scored at hospital discharge, a variable time frame. In fact, mortality was not different among users of newer and classical AED only, and the different likelihood of return to baseline may simply reflect the need for a longer convalescence after a severe SE episode, even after consideration of two solid SE predictors. Our study has some other potential limitations. A selection bias may exist because it considers only patients from a university hospital; however, most SE patients are treated in institutions with availability of ICU and neurologists. Due to its retrospective design, the analysis does not allow us to know whether patients were already receiving the assessed treatements before admission, or if these were initiated de novo at hospital during the seizure. Finally, we had no information about treatment side-effects.

In conclusion, despite some increase in the prescription of newer AED for SE treatment over the past few years, this study does not support an improved prognosis following their prescription, if considered as a group, and corroborates the aforementioned finding on the lack of superiority of levetiracetam as compared to valproate or phenytoin (Alvarez et al, 2011). Since newer AED are more expensive than classical ones, the marketing efforts aimed at their increased use in SE should rely on well-designed, prospective assessments showing their superiority (at least regarding side effects). Such studies would be of great importance in this clinical setting (Shorvon \& Ferlisi, 2011; Rossetti \& Lowenstein, 2011). 


\section{References}

Alacqua M, Trifirò G, Spina E, Moretti S, Tari DU, Bramanti P, et al. Newer and older antiepileptic drug use in Southern Italy: a population-based study during the years 2003-2005. Epilepsy Res. 2009 juill;85(1):107-13.

Alvarez V, Januel J-M, Burnand B, Rossetti AO. Second-line status epilepticus treatment: comparison of phenytoin, valproate, and levetiracetam. Epilepsia. 2011 juill;52(7):1292-6.

Berning S, Boesebeck F, van Baalen A, Kellinghaus C. Intravenous levetiracetam as treatment for status epilepticus. J. Neurol. 2009 oct;256(10):1634-42.

Beydoun A. Monotherapy trials of new antiepileptic drugs. Epilepsia. 1997;38 Suppl 9:S2131.

Chin RFM, Neville BGR, Scott RC. A systematic review of the epidemiology of status epilepticus. Eur. J. Neurol. 2004 déc;11(12):800-10.

Claassen J, Lokin JK, Fitzsimmons B-FM, Mendelsohn FA, Mayer SA. Predictors of functional disability and mortality after status epilepticus. Neurology. 2002 janv 8;58(1):13942.

Hsia Y, Neubert A, Sturkenboom MCJM, Murray ML, Verhamme KMC, Sen F, et al. Comparison of antiepileptic drug prescribing in children in three European countries. Epilepsia. 2010 mai;51(5):789-96.

Kellinghaus C, Berning S, Immisch I, Larch J, Rosenow F, Rossetti AO, et al. Intravenous lacosamide for treatment of status epilepticus. Acta Neurol. Scand. 2011 févr;123(2):137-41.

Knake S, Gruener J, Hattemer K, Klein KM, Bauer S, Oertel WH, et al. Intravenous levetiracetam in the treatment of benzodiazepine refractory status epilepticus. J. Neurol. Neurosurg. Psychiatr. 2008 mai;79(5):588-9.

Kwan P, Brodie MJ. Clinical trials of antiepileptic medications in newly diagnosed patients with epilepsy. Neurology. 2003 juin 10;60(11 Suppl 4):S2-12.

Logroscino G, Hesdorffer DC, Cascino G, Annegers JF, Hauser WA. Short-term mortality after a first episode of status epilepticus. Epilepsia. 1997 déc;38(12):1344-9.

Lowenstein DH. Status epilepticus: an overview of the clinical problem. Epilepsia. 1999;40 Suppl 1:S3-8; discussion S21-2.

Lowenstein DH, Alldredge BK. Status epilepticus. N. Engl. J. Med. 1998 avr 2;338(14):9706.

Marson AG, Al-Kharusi AM, Alwaidh M, Appleton R, Baker GA, Chadwick DW, et al. The SANAD study of effectiveness of valproate, lamotrigine, or topiramate for generalised and unclassifiable epilepsy: an unblinded randomised controlled trial. Lancet. 2007 mars 24;369(9566):1016-26.

Marson AG, Al-Kharusi AM, Alwaidh M, Appleton R, Baker GA, Chadwick DW, et al. The 
SANAD study of effectiveness of carbamazepine, gabapentin, lamotrigine, oxcarbazepine, or topiramate for treatment of partial epilepsy: an unblinded randomised controlled trial. Lancet. 2007 mars 24;369(9566):1000-15.

Meierkord H, Boon P, Engelsen B, Göcke K, Shorvon S, Tinuper P, et al. EFNS guideline on the management of status epilepticus in adults. Eur. J. Neurol. 2010 mars;17(3):348-55.

Novy J, Logroscino G, Rossetti AO. Refractory status epilepticus: a prospective observational study. Epilepsia. 2010 févr;51(2):251-6.

Novy J, Rossetti AO. Oral pregabalin as an add-on treatment for status epilepticus. Epilepsia. 2010 oct;51(10):2207-10.

Pugh MJV, Van Cott AC, Cramer JA, Knoefel JE, Amuan ME, Tabares J, et al. Trends in antiepileptic drug prescribing for older patients with new-onset epilepsy: 2000-2004. Neurology. 2008 mai 27;70(22 Pt 2):2171-8.

Rossetti AO, Hurwitz S, Logroscino G, Bromfield EB. Prognosis of status epilepticus: role of aetiology, age, and consciousness impairment at presentation. J. Neurol. Neurosurg. Psychiatr. 2006 mai;77(5):611-5.

Rossetti AO, Bromfield EB. Determinants of success in the use of oral levetiracetam in status epilepticus. Epilepsy Behav. 2006 mai;8(3):651-4.

Rossetti AO, Logroscino G, Milligan TA, Michaelides C, Ruffieux C, Bromfield EB. Status Epilepticus Severity Score (STESS): a tool to orient early treatment strategy. J. Neurol. 2008 oct;255(10):1561-6.

Rossetti AO, Lowenstein DH. Management of refractory status epilepticus in adults: still more questions than answers. Lancet Neurol. 2011 oct;10(10):922-30.

Rüegg S, Naegelin Y, Hardmeier M, Winkler DT, Marsch S, Fuhr P. Intravenous levetiracetam: treatment experience with the first 50 critically ill patients. Epilepsy Behav. 2008 avr;12(3):477-80.

Savica R, Beghi E, Mazzaglia G, Innocenti F, Brignoli O, Cricelli C, et al. Prescribing patterns of antiepileptic drugs in Italy: a nationwide population-based study in the years 20002005. Eur. J. Neurol. 2007 déc;14(12):1317-21.

Shneker BF, Fountain NB. Assessment of acute morbidity and mortality in nonconvulsive status epilepticus. Neurology. 2003 oct 28;61(8):1066-73.

Shorvon S. The management of status epilepticus. J. Neurol. Neurosurg. Psychiatr. 2001 juin;70 Suppl 2:II22-7.

Shorvon S. The treatment of status epilepticus. Curr. Opin. Neurol. 2011 avr;24(2):165-70.

Shorvon S, Ferlisi M. The treatment of super-refractory status epilepticus: a critical review of available therapies and a clinical treatment protocol. Brain. 2011 oct;134(Pt 10):2802-18.

Stojanova V, Rossetti AO. Oral topiramate as an add-on treatment for refractory status epilepticus. Acta Neurologica Scandinavica [Internet]. 2011 juin 29 [cité 2011 nov 
20];Available from: http://www.ncbi.nlm.nih.gov/pubmed/21711264

Swisher CB, Doreswamy M, Gingrich KJ, Vredenburgh JJ, Kolls BJ. Phenytoin, Levetiracetam, and Pregabalin in the Acute Management of Refractory Status Epilepticus in Patients with Brain Tumors. Neurocritical Care [Internet]. 2011 sept 1 [cité 2011 nov 20];Available from: http://www.ncbi.nlm.nih.gov/pubmed/21882056

Towne AR, Garnett LK, Waterhouse EJ, Morton LD, DeLorenzo RJ. The use of topiramate in refractory status epilepticus. Neurology. 2003 janv 28;60(2):332-4.

Towne AR, Pellock JM, Ko D, DeLorenzo RJ. Determinants of mortality in status epilepticus. Epilepsia. 1994 févr;35(1):27-34.

Trinka E, Dobesberger J. New treatment options in status epilepticus: a critical review on intravenous levetiracetam. Ther Adv Neurol Disord. 2009 mars;2(2):79-91.

Tsai M-H, Chuang Y-C, Chang H-W, Chang W-N, Lai S-L, Huang C-R, et al. Factors predictive of outcome in patients with de novo status epilepticus. QJM. 2009 janv;102(1):5762.

Vazquez B. Monotherapy in epilepsy: role of the newer antiepileptic drugs. Arch. Neurol. 2004 sept;61(9):1361-5. 
Table 1. Treatment of SE episodes stratified according to two periods of time.

\begin{tabular}{|c|c|c|c|}
\hline & $\begin{array}{c}\text { Classical AED } \\
\text { only }\end{array}$ & Newer AED & p-value (test) \\
\cline { 1 - 3 } April 2006- September 2007 & $59(69.41 \%)$ & $26(30.59 \%)$ & \multirow{2}{*}{$0.060\left(\chi^{2}\right)$} \\
\cline { 1 - 3 } October 2007- September 2010 & $140(57.85 \%)$ & $102(42.15 \%)$ & \\
\hline
\end{tabular}

Table 2. Prognosis according to two periods of time.

\begin{tabular}{|l|c|c|c|c|}
\hline & Return to baseline & Disabled & Dead & p-value \\
\hline April 2006- September 2007 & $42(49.41 \%)$ & $28(32.94 \%)$ & $15(17.65 \%)$ & \multirow{2}{*}{$0.303\left(\chi^{2}\right)$} \\
\cline { 1 - 4 } October 2007- September 2010 & $127(52.48 \%)$ & $88(36.36 \%)$ & $27(11.16 \%)$ & \\
\hline
\end{tabular}

Table 3. Prognosis regarding to the medication in the whole study period

\begin{tabular}{|l|c|c|c|c|}
\hline $042006-092010$ & Return to baseline & Disabled & Dead & p-value \\
\hline Classical AEDonly & $127(63.81 \%)$ & $54(27.14 \%)$ & $18(9.04 \%)$ & $<0.001\left(\chi^{2}\right)$ \\
\hline Newer AED & $42(32.81 \%)$ & $62(48.43 \%)$ & $24(18.75 \%)$ & \\
\hline
\end{tabular}

Table 4. Prevalence of potential fatal etiology and STESS (status epilepticus severity score) regarding to the use of medication in the whole study period

\begin{tabular}{|l|c|c|c|c|}
\hline & No pot. fatal etiology & Pot. fatal etiology & STESS 0-2 & STESS 3-6 \\
\hline Classical AED only & $129(64.82 \%)$ & $70(35.18 \%)$ & $107(53.77 \%)$ & $92(46.23 \%)$ \\
\hline Newer AED & $59(46.1 \%)$ & $69(53.9 \%)$ & $55(42.97 \%)$ & $73(57.03 \%)$ \\
\hline p-value & \multicolumn{2}{|c|}{$0.001\left(\chi^{2}\right)$} & \multicolumn{2}{|c|}{$0.057\left(\chi^{2}\right)$} \\
\hline
\end{tabular}

Table 5. Multivariable logistic regression models using risk of lack to return to baseline clinical conditions at hospital discharge, or mortality, as outcome. Results are given as OR (95\% CI).

\begin{tabular}{|l|c|c|}
\hline & Lack of return to baseline & Mortality \\
\hline Use of newer AED & $3.23(1.88-5.52), \mathrm{p}<0.001$ & $1.60(0.80-3.21), \mathrm{p}=0.18$ \\
\hline STESS 3-6 & $4.08(2.42-6.89), \mathrm{p}<0.001$ & $4.11(1.80-9.38), \mathrm{p}<0.001$ \\
\hline Potentially fatal etiology & $5.14(3.03-8.73), \mathrm{p}<0.001$ & $4.32(2.00-9.33), \mathrm{p}<0.001$ \\
\hline Goodnes of fit $\left(\chi^{2}\right)$ & 0.23 & 0.60 \\
\hline
\end{tabular}

AED = antiepileptic drugs; STESS = Status Epilepticus Severity Score 